\title{
Mammary hamartoma: is ultrasound-guided vacuum-assisted breast biopsy sufficient for its treatment?
}

\author{
Huayu Hu ${ }^{1,2}$, Mengke Zhang ${ }^{3}$, Yuan Liu ${ }^{3}, \mathrm{Xi}-\mathrm{Ru} \mathrm{Li}^{2}$, Gang Liu ${ }^{4}$, Zhili Wang ${ }^{3}$ \\ ${ }^{1}$ School of Medicine, Nankai University, Tianjin, China; ${ }^{2}$ Department of General Surgery, ${ }^{3}$ Department of Ultrasound, ${ }^{4}$ Department of Radiology, \\ Chinese People's Liberation Army General Hospital, Beijing, China \\ Contributions: (I) Conception and design: H Hu, Z Wang; (II) Administrative support: Z Wang, XR Li, G Liu; (III) Provision of study materials or \\ patients: All authors; (IV) Collection and assembly of data: Z Wang, Y Liu; (V) Data analysis and interpretation: H Hu, M Zhang; (VI) Manuscript \\ writing: All authors; Final approval of manuscript: All authors. \\ Correspondence to: Zhili Wang. Department of Ultrasound, Chinese People's Liberation Army General Hospital, 28 Fuxing Road, Beijing 100853, \\ China. Email: wzllg@sina.com; Gang Liu. Department of Radiology, Chinese People’s Liberation Army General Hospital, 28 Fuxing Road, Beijing \\ 100853, China. Email: lgwzl@126.com; Xi-Ru Li. Department of General Surgery, Chinese People's Liberation Army General Hospital, 28 Fuxing \\ Road, Beijing 100853, China. Email: 2468li@sina.com.
}

\begin{abstract}
Background: Mammary hamartomas were mostly benign tumors with rare rate of recurrence and malignant transformation. Ultrasound (US)-guided vacuum-assisted breast biopsy (VABB) has been reported sufficiently safe in treating many breast benign tumors but remained undefined in mammary hamartoma for its usual underdiagnosis in US. Thus, this study aims to evaluate the efficiency of US-guided VABB in treating mammary hamartomas.
\end{abstract}

Methods: From May 2015 to March 2019, 3,388 lesions of 2,534 patients underwent percutaneous USguided VABB, among which 31 mammary hamartomas proved by pathology were included in this study. Patients were followed up by US three, six and twelve months later, then at 1-year intervals. Lesions were classified to analyze the possible factors associated with excision rate, bleeding volume and complications.

Results: Of the 31 patients, recurrence was seen in 1 case in 1 year after the procedure and complete excision rate was $96.8 \%$ (30/31). The bleeding volume ranged from 1 to $15 \mathrm{~mL}$ (mean number \pm standard deviation, $6.5 \pm 3.4 \mathrm{~mL}$ ) and significant statistical differences were detected in patient age and the largest diameter of lesions. The main complications included pain $(22.6 \%)$, hematomas $(9.7 \%)$ and ecchymosis (3.2\%).

Conclusions: US-guided VABB ensures an outstanding complete excision rate and provides an alternative solution to treat mammary hamartomas.

Keywords: Ultrasound-guided vacuum-assisted breast biopsy (VABB); mammary hamartoma; complete excision rate

Submitted Apr 02, 2020. Accepted for publication Aug 17, 2020.

doi: $10.21037 / g s-20-437$

View this article at: http://dx.doi.org/10.21037/gs-20-437

\section{Introduction}

Mammary hamartomas, also referred to as lipofibroadenomas, adenolipomas, and fibroadenolipomas, were rare benign lesions accounting for approximately $0.7-4.8 \%$ in all breast benign tumors $(1,2)$. Mammary hamartoma were initially described by Arrigoni in 1971 (3), characterized by well-circumscribed oval masses composed of different proportions of fat, fibrous tissue and glandular epithelial components. They were mostly soft, round and impalpable masses without any clinical symptoms. Rare giant mammary hamartomas were reported $(4,5)$. Although hamartomas were almost benign lesions, a few rare cases associated with malignant invasive ductal or lobular carcinoma have 
been reported (6-9). Additionally, recurrence was also an uncommon incident, about $8 \%$ in all cases (10).

In previous cases, mammary hamartomas were primarily first diagnosed by mammography according to pseudocapsule and internal elements like water density. Once hamartoma was detected, follow-up observation or surgical resection were two measures provided. It's used to be thought that mammary hamartomas were underdiagnosed in ultrasound (US) because doctors had difficulty in distinguishing them from normal breast images. Actually US imaging has made progress in recent years. The appearance of shear wave elastography and contrast-enhanced ultrasonography provided more accurate diagnosis for hamartoma (11). Doctors could diagnose mammary hamartoma based on some typical characteristics in US, which made US-guided vacuum-assisted breast biopsy (VABB) become an alternative for those who refused regular check and surgical operation.

US-guided VABB has proved to be a safe and minimally invasive surgical substitute for most breast benign tumors, while in other special pathology category, a second surgical resection was necessary (12). However, there have been no systematic studies on the diagnosis and treatment of mammary hamartoma under US-guided VABB, particularly on its complete excision and recurrence.

In this study, we analyzed 31 cases diagnosed as mammary hamartoma via US and treated by US-guided $\mathrm{VABB}$, aiming at evaluating whether VABB was sufficient for the treatment of mammary hamartoma.

We present the following article in accordance with the STROBE reporting checklist (available at http://dx.doi. org/10.21037/gs-20-437).

\section{Methods}

\section{Patients and lesions}

The study was conducted in accordance with the Declaration of Helsinki (as revised in 2013). The study was approved by Medical Ethics Committee of Chinese PLA General Hospital (No. S2020-336-01) and informed consent was taken from all the patients. From May 2015 to March 2019, 3,388 lesions of 2,534 patients underwent percutaneous US-guided vacuum assisted breast biopsy, among which 33 mammary hamartomas proved by pathology were selected. Two patients were excluded because of their loss to follow up and the remaining 31 patients were included in this study. All of them were female patients without family history of breast cancer. Ages of the patients ranged from 25 to 54 years (mean age \pm standard deviation, $40.2 \pm 7.8$ years). There were 27 premenopausal patients and 4 postmenopausal patients.

All the lesions were classified on the basis of Breast Imaging Reporting and Data System (BI-RADS), 30 lesions were classified category 3 and one lesion was category $4 a$. In terms of location, 10 lesions were in the inner quadrant and 21 lesions were in the outer quadrant. The largest diameters of the lesions were 1.4 to $5.5 \mathrm{~cm}$ (mean size \pm standard deviation, $3.2 \pm 1.0 \mathrm{~cm}$ ). All the tumors didn't cause obvious symptoms, 22 lesions were palpable while 9 lesions were impalpable. US-guided VABB resections were recommended when patients suffered severe anxiety about the lesions or the size was increasing.

US-guided VABB couldn't be performed to BI-RADS category 5 or 6 lesions. Other contraindication included local anesthetics allergy, abnormal ECG, coagulation disorder and the use of anticoagulants (aspirin and coumarone) within seven days before operation.

\section{US examination}

US examinations were conducted by two radiologists Zhili Wang and Yuan Liu, sophisticated in breast US with 18 years' experience, using the iU22 ultrasound system (Philips Medical Systems, Andover, Massachusetts) with a L12-5 linear array probe. All the patients were in lateral decubitus position with their ipsilateral arms raised and hands under the head. Radial exploration approach was used to ensure that none of lesions were missed. Once found, the lesion would be reconfirmed in the corresponding vertical section and a third diameter would be measured. Meanwhile, the following information about the lesion was recorded: position, the largest diameter and size, margin, internal echogenicity, blood flow signal, BI-RADS category. The blood flow was categorized into level 0, 1, 2 to represent no, little and rich intralesional blood flow.

\section{Anesthesia}

A $10 \mathrm{~mL}$ injection syringe was filled with $5 \mathrm{~mL}$ of $1 \%$ lidocaine, $4 \mathrm{~mL}$ hemocoagulase purified from Bothrops atrox and $1 \mathrm{~mL}$ adrenaline hydrochloride. The final concentration of adrenaline in the mixture was 1:100,000. Then the mixture was injected into the cutaneous layer and structure around the lesion, including subcutaneous fat 

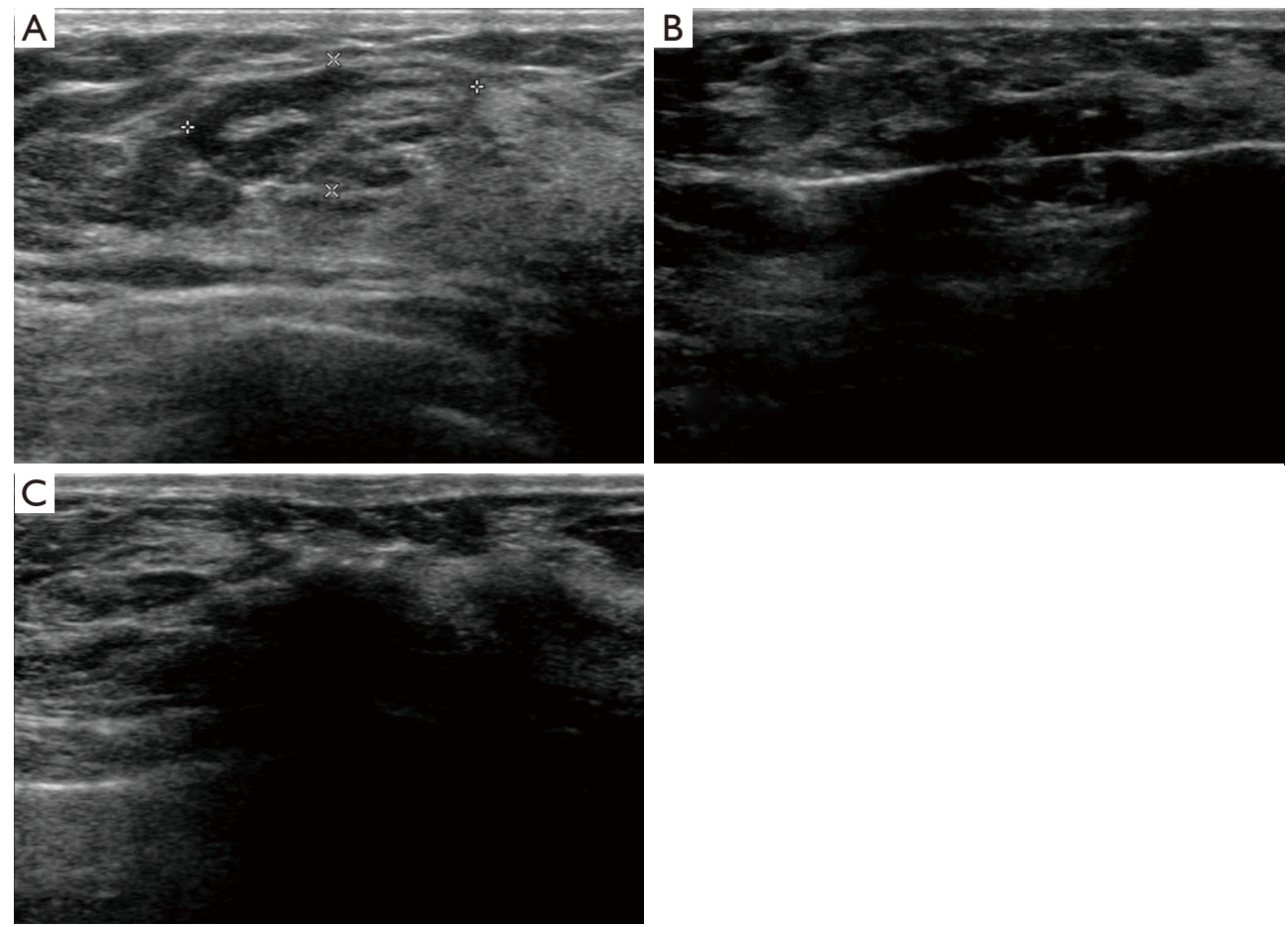

Figure 1 A US-guided VABB procedure performed in a 43-year-old woman. (A) A well-circumscribed lesion with a pseudocapsule in US image, which was proved as mammary hamartoma pathologically. (B) A rotation needle was inserted into the lesion. (C) A 360-degree extended resection was performed. VABB, vacuum-assisted breast biopsy; US, ultrasound.

tissue and retromammary space, to ensure both anesthesia and hemostasis effect. For lesions adjacent to pectoralis major and skin, more fluid injection around the mass was indispensable in order to increase the safe distance.

\section{US-guided VABB procedure}

The 7 -gauge EnCor ${ }^{\circledR}$ system (EnCor ${ }^{\circledR}$ MR, SenoRx, Allso Viejo, CA, USA) was used for the US-guided VABB procedure performed by a certificated radiologist Zhili Wang. After the US localization and anesthesia, a scalpel blade was held to cut a $3 \mathrm{~mm}$ incision on the edge of mammary areola, from where the rotation needle was inserted into the lesion and the excised tissue was transported to the biopsy blanket assisted by vacuum device. After the whole lesion was removed, a 360-degree extended resection was performed to eliminate residences (Figure 1). The whole procedure was monitored by US. Samples excised from each lesion were 5 to 96 samples (mean sample \pm standard deviation, $29.1 \pm 19.2$ sample). All the samples were sent for pathological examination (Figure 2).

The assistant compressed the surgical area for 15 minutes until the bleeding and hematoma were controlled. A second US was necessary to assess whether there was any residuals or low echogenicity blood aggregation. If any hematoma, the area needed to be squeezed with gauze until the low echogenicity size could be no more decreased. Elastic bandage was covered tightly around the breast for 24-48 hours.

Bleeding volume was the sum of the blood suctioned by vacuum and squeezed by gauze. The latter was estimated according to area of blood-soaked gauze: dye the gauze with $10 \mathrm{~mL}$ blood to calculate the dye area and estimate other gauzes.

\section{Follow-up}

All the patients were asked to the hospital for a US examination 3 days after the procedure to make sure no serious complications happened. Subsequently, they would be followed up by US three, six and twelve months later, then at 1-year intervals. The main focuses during the follow-up were the recovery of hematoma or bruising, the healing of incision and any new masses reappearing in situ. 


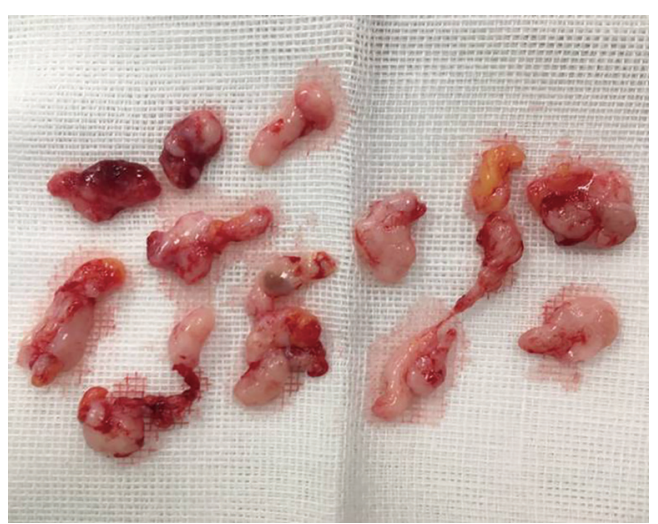

Figure 2 Samples of a lesion resected by US-guided VABB. VABB, vacuum-assisted breast biopsy; US, ultrasound.

\section{Statistical analysis}

All data statistics were analyzed by SPSS 23.0, standard version (SPSS Inc., Chicago, IL). Continuous variables were described as mean $\pm \mathrm{SD}$. The $t$-test was used for data from two different groups confirmed as normal distribution and homogeneity of variance, otherwise a Rank test was used. Variance analysis was used for mean comparison among multiple groups. The $\chi^{2}$ test or Fisher's exact test were used for the comparison of dichotomous variables in different subgroups. $\mathrm{P}$ value $<0.05$ was considered significant statistical difference.

\section{Results}

\section{Pathologic features}

All the 31 cases in this study were mammary hamartomas pathologically (Table 1, Figure 3). Epithelial hyperplasia was present in 4 cases. Seventeen cases were diagnosed as adenosis accompanied. Furthermore, one case was associated with sclerosing adenosis. Microcalcification, pseudoangiomatous hyperplasia, myoid hamartoma and malignancy were not detected in all cases.

\section{Complete excision rate}

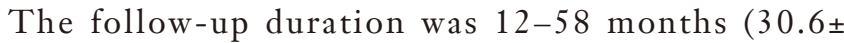
22.7 months). Connections between features of lesions and excision rate were summarized in Table 2. In 31 cases, only one patient was found a hypoechoic mass in the original position 1 year later, which was believed to be a recurrence that had been ruled out for postoperative changes. The
Table 1 Clinicopathologic features

\begin{tabular}{lc}
\hline Features & Number \\
\hline Total hamartoma cases & 31 \\
Median age & $40.2[25-54]$ \\
Microcalcification & 0 \\
Epithelial hyperplasia & 4 \\
Breast adenosis & 17 \\
Sclerosing adenosis & 1 \\
Pseudoangiomatous hyperplasia & 0 \\
Myoid hamartoma & 0 \\
Malignancy & 0 \\
\hline
\end{tabular}

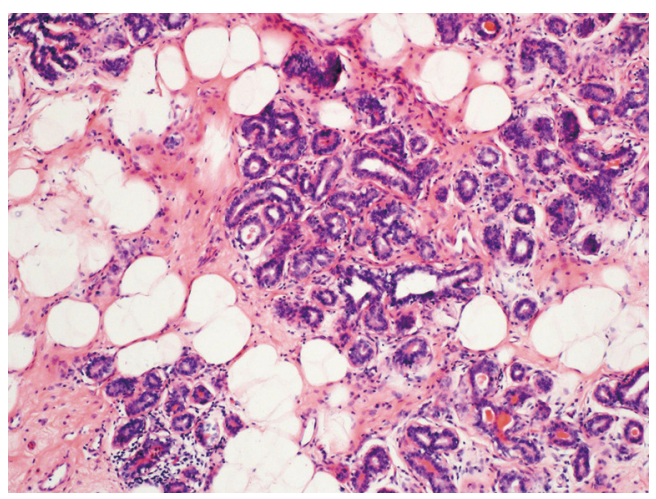

Figure 3 Mammary hamartoma with normal ductal lobular unit and fat tissue, hematoxylin eosin $(\mathrm{H} \& \mathrm{E}), \times 100$.

mass was followed up closely for its small size $(4 \mathrm{~mm})$. Rate of complete excision was 96.8 percent (30/31). Age of patient, palpability of lesion, lesion size and border, blood flow, internal echogenicity didn't have significant statistical differences in each subgroup. No malignant transformation occurred in all lesions.

\section{Bleeding volume}

The method for estimating the amount of bleeding was described in the previous paragraph. The bleeding volume ranged from 1 to $15 \mathrm{~mL}$ (mean number \pm standard deviation, $6.5 \pm 3.4 \mathrm{~mL}$ ). Among all the possible factors, patient age and the largest diameter had significant statistical differences (Table 3). No significant difference was seen in palpability of lesion, lesion border, blood flow and internal echogenicity. 
Table 2 Excision rate in divided groups

\begin{tabular}{|c|c|c|}
\hline Lesion features & Excision rate & $P$ value \\
\hline Age & & 1.00 \\
\hline$<40$ & $100 \%(13 / 13)$ & \\
\hline$\geq 40$ & $94.4 \%(17 / 18)$ & \\
\hline Palpable & & 0.29 \\
\hline Yes & $100 \%(22 / 22)$ & \\
\hline No & $88.9 \%(8 / 9)$ & \\
\hline Largest diameter & & 1.00 \\
\hline$<3 \mathrm{~cm}$ & $100 \%(14 / 14)$ & \\
\hline$\geq 3 \mathrm{~cm}$ & $94.1 \%(16 / 17)$ & \\
\hline Lesion edge & & 1.00 \\
\hline Circumscribed & $95.2 \%(20 / 21)$ & \\
\hline Noncircumscribed & $100 \%(10 / 10)$ & \\
\hline Blood flow & & 1.00 \\
\hline Level 0 & $100 \%(11 / 11)$ & \\
\hline Level 1 & $93.8 \%(15 / 16)$ & \\
\hline Level 2 & $100 \%(4 / 4)$ & \\
\hline Internal echogenicity & & 1.00 \\
\hline Heterogeneous & $95.5 \%(21 / 22)$ & \\
\hline Dominant hypoechoic & $100 \%(6 / 6)$ & \\
\hline Dominant hyperechoic & $100 \%(3 / 3)$ & \\
\hline
\end{tabular}

\section{Complications}

The main complications included pain (22.6\%), hematomas $(9.7 \%)$ and ecchymosis $(3.2 \%)$ mostly within five days after the procedure. Seven patients suffered pain on operation day and recovered two days later. Hematomas were found in three patients. The largest area was about $5 \mathrm{~cm} \times 5 \mathrm{~cm}$ and disappeared in several months, which was considered linked with lesion size (5.5 $\mathrm{cm}$ in largest diameter). Ecchymosis was seen in only one patient and relived gradually. No infection events were found in all patients.

\section{Discussion}

Mammary hamartomas were considered rare with a low incidence in previous studies (1,9), mostly diagnosed in mammography and demonstrated by pathology. In US images, mammary hamartomas were called "breast within
Table 3 Bleeding volume in divided groups

\begin{tabular}{|c|c|c|}
\hline Lesion features & $\begin{array}{l}\text { Bleeding volume } \\
\text { (mean } \pm \mathrm{SD} \mathrm{mL} \text { ) }\end{array}$ & $P$ value \\
\hline Age & & $<0.01$ \\
\hline$<40$ & $4.5 \pm 2.5$ & \\
\hline$\geq 40$ & $7.9 \pm 3.2$ & \\
\hline Palpable & & 0.45 \\
\hline Yes & $6.2 \pm 3.2$ & \\
\hline No & $7.2 \pm 3.9$ & \\
\hline Largest diameter & & 0.01 \\
\hline$<3 \mathrm{~cm}$ & $4.4 \pm 1.9$ & \\
\hline$\geq 3 \mathrm{~cm}$ & $8.2 \pm 3.3$ & \\
\hline Lesion edge & & 0.18 \\
\hline Circumscribed & $7.0 \pm 3.0$ & \\
\hline Noncircumscribed & $5.3 \pm 3.9$ & \\
\hline Blood flow & & 0.41 \\
\hline Level 0 & $7.2 \pm 3.6$ & \\
\hline Level 1 & $6.5 \pm 3.4$ & \\
\hline Level 2 & $4.5 \pm 2.6$ & \\
\hline Internal echogenicity & & 0.64 \\
\hline Heterogeneous & $6.5 \pm 3.6$ & \\
\hline Dominant hypoechoic & $5.7 \pm 1.5$ & \\
\hline Dominant hyperechoic & $8.0 \pm 4.6$ & \\
\hline
\end{tabular}

a breast" for their normal structures. These structures were thought to be a result of abnormal development of embryo but still comprised of normal breast tissues. With the development of US technique and increasing experience on these diseases, radiologists could diagnose mammary hamartoma depend on some special characteristics in US images. Hyperechoic pseudocapsule, heterogeneous internal echogenicity, multiple stratification or target sign were typical signs of hamartomas in breast US images (11). Actually mammary hamartomas were found mostly in US examination in our institution.

Pathologically, epithelial changes, lobular hyperplasia and adenosis were observed in our study, as was confirmed in other studies $(9,13)$. Albawardi et al. found mammary hamartomas associated with flat epithelial atypia (14). In this study, epithelial hyperplasia was seen in 4 cases, but not atypical ones. Sclerosing adenosis, also called radial 
scar, was found in one case, which was present as well in the study of Türkyllmaz (9). Microcalcifications suggested a certain probability of potential malignancy (15). Daya et al. and Park et al. reported calcification in $25 \%$ cases in their studies $(10,16)$. Pseudoangiomatous hyperplasia was thought an uncommon histological characteristic in a fraction of cases $(13,17)$. Herein, no calcification, malignancy and pseudoangiomatous hyperplasia were seen in our study. Some case reports $(13,18)$ have analyzed features of myoid hamartoma, an extremely rare subtype of mammary hamartoma. And myoid hamartoma wasn't seen in this study.

In this study, US-guided VABB proved to be a sufficient means to treat mammary hamartomas, with only one lesion recurred and other lesions completely resected. Very seldom, mammary hamartomas recurred after the surgical resection. Daya et al. reported a $8 \%$ postoperative recurrence $(2 / 25)$ with the lesion sizes ranging from 1 to $7 \mathrm{~cm}$ in maximum dimension (mean, $3.9 \mathrm{~cm}$ ) (10). The recurrence and complete resection rate of VABB in this study was similar with traditional surgical resection. To pursue a perfect removal, we performed a 360-degree expanding excision right after there have been no residences of masses in US images. Additionally, most mammary hamartomas in our study were well-circumscribed, making it easy for the operator to judge the lesion edge and gain a negative margin (19). Patients or lesions were divided into subgroups and no significant difference was seen, which meant US-guided VABB had an outstanding therapeutic effect in each group.

It's worth mentioning that US-guided VABB had an excellent bleeding control to treat mammary hamartomas, with a medial volume of $7 \mathrm{~mL}$, much less than traditional surgical operation. To attain a low bleeding, adrenaline hydrochloride and hemocoagulase Bothrops atrox were injected around target lesions. Adrenaline hydrochloride could cause local vasoconstriction for bleeding reduction, simultaneously slow down the absorption of lidocaine to relieve pain. Hydrochloride has been diluted to a safe concentration that wouldn't cause the fluctuation of blood pressure and heart rate. Furthermore, effective compression and squeeze on the operation site facilitated the hemostasis. Significant differences were seen in patient age and size in terms of bleeding. Elderly patients (age $\geq 40$ years) with larger masses (largest diameter $\geq 3 \mathrm{~cm}$ ) might tend to have more bleeding. Those people needed to be paid more attention during the procedure.

Very few complications were found in our study and no severe complication events occurred. It's reported that hematoma was the most common complication after VABB, accounting for $9 \%$ (20), which was similar to our data (9.7\%). Hydrochloride, hemocoagulase Bothrops atrox and adequate compression played crucial roles in controlling hematomas and ecchymosis. Owing to careful preoperative examinations (ECG, coagulation test, detailed allergy history), patients with contraindications were excluded from the operation. There were no adverse events like serious vasovagal reflex and anaphylaxis.

There were no cases linked with malignant transformation or atypical hyperplasia in this study. Previously some case reports claimed to discover mammary hamartomas occurred accompany with carcinoma, invasive or in situ $(6-9,21)$. Tse et al. concluded that carcinoma was more likely to occur in lesions with a size more than $5.8 \mathrm{~cm}$ (22). It's still under discussion whether mammary hamartoma conducted a malignant transformation or a carcinoma grew just as a neighbor and then into the hamartoma. Microcalcification is acknowledged to be a crucial indicator of malignant modification and sensitive to be observed in mammography (23). Given the denser breast tissue, US examination is generally the primary tool for detecting breast diseases in China. Because no microcalcifications were found pathologically in our study, we didn't conduct mammography examinations.

The subsequent procedure after VABB was determined by the pathological results. If any dubious points such as atypical hyperplasia or phyllodes tumors existed, a second surgery would be performed to achieve total excision. In this study, no potential underdiagnosed lesions were found, so we thought that US follow-up was enough. A lesion was regarded as completely resected when there was no residue in situ under US surveillance.

The highlight of our study is that VABB can treat mammary hamartoma as an alternative to traditional surgery, especially for larger ones (diameter $\geq 3 \mathrm{~cm}$ ). The remarkable complete resection rate may be credited to its unique structure. Hamartoma is composed of normal distorted tissue, which contributes to its low recurrence compared with other breast tumors. Therefore, the size limit for VABB can be more flexible for hamartoma.

Our study also has limitations. Firstly, although patients included in this study are more than those in some recent studies on mammary hamartoma, the overall number is still small. Mammary hamartoma is an uncommon breast disease with a rare incidence, data in a single center is general limited. What's more, pathological features of mammary 
hamartomas are not easily identifiable. So hamartomas could be categorized as fibroadenomas or a pile of normal breast tissue pathologically if clinical information isn't provided (13). Therefore the true incidence may be higher in the real world. Secondly, there are too few recurrences in this study to investigate deeply the association between possible factors and tumor recurrences. Thus, multicenter researches on mammary hamartoma may be needed in further studies.

In conclusion, US-guided VABB ensures an outstanding complete excision rate and provides an alternative solution to treat mammary hamartomas.

\section{Acknowledgments}

Funding: This work was supported by the National Natural Science Foundation (grant number: 81771832), Military Top project of Youth Training for Medical Science and Technology (grant number: 19QNP071), and Military Health-care Topic (grant number: 17BJZ34).

\section{Footnote}

Reporting Checklist: The authors have completed the STROBE reporting checklist. Available at http://dx.doi. org/10.21037/gs-20-437

Data Sharing Statement: Available at http://dx.doi. org/10.21037/gs-20-437

Peer Review File: Available at http://dx.doi.org/10.21037/gs20-437

Conflicts of Interest: All authors have completed the ICMJE uniform disclosure form (available at http://dx.doi. org/10.21037/gs-20-437). XRL served as the unpaid Editors-in-Chief of Gland Surgery from May 2017 to April 2022. ZW served as the unpaid editorial board member of Gland Surgery from April 2019 to March 2021. The other authors have no conflicts of interest to declare.

Ethical Statement: The authors are accountable for all aspects of the work in ensuring that questions related to the accuracy or integrity of any part of the work are appropriately investigated and resolved. The study was conducted in accordance with the Declaration of Helsinki (as revised in 2013). The study was approved by Medical Ethics Committee of Chinese PLA General Hospital (No.
S2020-336-01) and informed consent was taken from all the patients.

Open Access Statement: This is an Open Access article distributed in accordance with the Creative Commons Attribution-NonCommercial-NoDerivs 4.0 International License (CC BY-NC-ND 4.0), which permits the noncommercial replication and distribution of the article with the strict proviso that no changes or edits are made and the original work is properly cited (including links to both the formal publication through the relevant DOI and the license). See: https://creativecommons.org/licenses/by-nc-nd/4.0/.

\section{References}

1. Charpin C, Mathoulin MP, Andrac L, et al. Reappraisal of breast hamartomas. A morphological study of 41 cases. Pathol Res Pract 1994;190:362-71.

2. Ravakhah K, Javadi N, Simms R. Hamartoma of the breast in a man: first case report. Breast J 2001;7:266-8.

3. Arrigoni MG, Dockerty MB, Judd ES. The identification and treatment of mammary hamartoma. Surg Gynecol Obstet 1971;133:577-82.

4. Wang Z, He J. Giant breast hamartoma in a 41-year-old female: A case report and literature review. Oncol Lett 2015;10:3719-21.

5. Ghaedi Y, Howlett D. Giant left breast hamartoma in a 45-year-old woman. BMJ Case Rep 2018;2018:bcr2018226012.

6. Kemp TL, Kilgore MR, Javid SH. Invasive Ductal Carcinoma Arising within a Large Mammary Hamartoma. Breast J 2015;21:196-7.

7. Lambert J, Jerjir N, Casselman J, et al. Invasive lobular carcinoma arising in a hamartoma of the breast: a case report. Clin Breast Cancer 2015;15:e63-6.

8. Coyne J, Hobbs FM, Boggis C, et al. Lobular carcinoma in a mammary hamartoma. J Clin Pathol 1992;45:936-7.

9. Türkyılmaz Z, Aydin T, Yilmaz R, et al. Our 20-Year Institutional Experience with Surgical Approach for Breast Hamartomas. Eur J Breast Health 2019;15:171-5.

10. Daya D, Trus T, D'Souza TJ, et al. Hamartoma of the breast, an underrecognized breast lesion. A clinicopathologic and radiographic study of 25 cases. Am J Clin Pathol 1995;103:685-9.

11. Liu G, Wang ZL, Zhang MK, et al. Breast hamartoma: Ultrasound, elastosonographic, and contrast-enhanced ultrasound features. J Cancer Res Ther 2019;15:864-70.

12. Youk JH, Kim H, Kim EK, et al. Phyllodes tumor 
diagnosed after ultrasound-guided vacuum-assisted excision: should it be followed by surgical excision? Ultrasound Med Biol 2015;41:741-7.

13. Sevim Y, Kocaay AF, Eker T, et al. Breast hamartoma: a clinicopathologic analysis of 27 cases and a literature review. Clinics (Sao Paulo) 2014;69:515-23.

14. Albawardi AS, Al Sharri SM, Al Bashir M, et al. Flat epithelial atypia in a mammary hamartoma: case report \& clinicopathologic correlates. Int J Clin Exp Med 2016;9:4896-900.

15. Bonfiglio R, Scimeca M, Toschi N, et al. Radiological, Histological and Chemical Analysis of Breast Microcalcifications: Diagnostic Value and Biological Significance. J Mammary Gland Biol Neoplasia 2018;23:89-99.

16. Park SY, Oh KK, Kim EK, et al. Sonographic findings of breast hamartoma: emphasis on compressibility. Yonsei Med J 2003;44:847-54.

17. Herbert M, Schvimer M, Zehavi S, et al. Breast hamartoma: fine-needle aspiration cytologic finding. Cancer 2003;99:255-8.

Cite this article as: Hu H, Zhang M, Liu Y, Li XR, Liu G, Wang Z. Mammary hamartoma: is ultrasound-guided vacuumassisted breast biopsy sufficient for its treatment? Gland Surg 2020;9(5):1278-1285. doi: 10.21037/gs-20-437
18. Yu L, Yang W, Xu X, et al. Myoid harmatoma of the breast: clinicopathologic analysis of a rare tumor indicating occasional recurrence potential. Breast J 2011;17:322-4.

19. Chen Y, Tang L, Du Z, et al. Factors influencing the performance of a diagnostic model including contrastenhanced ultrasound in 1023 breast lesions: comparison with histopathology. Ann Transl Med 2019;7:647.

20. Fang M, Liu G, Luo G, et al. Feasibility and safety of image-guided vacuum-assisted breast biopsy: A PRISMAcompliant systematic review and meta-analysis of 20000 population from 36 longitudinal studies. Int Wound J 2019;16:1506-12.

21. Amir RA, Sheikh SS. Breast hamartoma: A report of 14 cases of an under-recognized and under-reported entity. Int J Surg Case Rep 2016;22:1-4.

22. Tse GM, Law BK, Ma TK, et al. Hamartoma of the breast: a clinicopathological review. J Clin Pathol 2002;55:951-4.

23. Fanizzi A, Basile TMA, Losurdo L, et al. A machine learning approach on multiscale texture analysis for breast microcalcification diagnosis. BMC Bioinformatics 2020;21:91. 